\title{
Probable Dates of Flex, Substantial Reduction in the Number of COVID-19 Positive Confirmed Cases and Fatalities in the State of Maharashtra (India)
}

B. L .ARORA ( $\sim$ brijarora@yahoo.com )

Formerly with A. R. S. D. COLLEGE (University of Delhi), DELHI, INDIA

\section{Research Article}

Keywords: COVID-19, Flex, Gauss error function, Positive cases, Fatalities

Posted Date: September 1st, 2020

DOI: https://doi.org/10.21203/rs.3.rs-68712/v1

License: (c) (i) This work is licensed under a Creative Commons Attribution 4.0 International License.

Read Full License 


\section{Abstract}

In this paper, we analyse the COVID-19 data of the number of confirmed positive infectious COVID-19 cases (I) in Maharashtra, the second most populous state of India, having a population of nearly 123 million - more than the population of most of the European countries. For this analysis, we use COVID-19 data for the period from April 1, 2020 to August 24, 2020 to find the flex in the I- $t$ curve, where the second derivative of the curve becomes negative. i.e. the date from which the rate of growth of the number of infections starts decreasing - or a peak occurs in the daily new COVID - 19 positive cases. Here I is the total number of cumulative COVID-19 positive cases and $t$ is the time (in days). The observed data are fitted by employing the Gauss error function formula

$a+b \operatorname{erf}(c x-d)$

(with four adjustable arbitrary parameters a, b, c, and d) following the prescriptions adopted by Ciufolini and Paolozzi [1] for the analysis of the COVID-19 data from Italy and China. The date of flex is found using data from April 1 to August 11, then the data from April 1 to August 12, and so on, till the data from April 1 to August 24. There is a variation in the dates of flex for these 14 sets of data; however, the date of flex converges to a definite date towards the later sets. We also calculate the standard deviation of these values to calculate the uncertainty in the expected date of flex. Using these parameter values, we also calculate the expected values of COVID-19 positive cases for future. From this data, we estimate the date(s) at which there is sufficient reduction in the number of new daily positive cases and the number of such cases are likely to increase by say, 1000 .

The data for the number of fatalities in the city is also fitted to a Gauss error function with four parameters of the above type and we estimate in this case also, the date of flex as well as the dates at which the number of fatalities reduces in proportion to sufficient reduction in the number of new COVID19 cases, which in this case is approximately 34 (for 1000 new infections). The data for the above analysis has been taken from the website covid19India.org and Aarogya Setu App [2] of the Government of India.

\section{Main Text}

The scourge of the devastating pandemic of COVID-19 has unequivocally proved to be a bane for the entire world, afflicting an enormously large number of people in all countries without exception. Ever since its inception, the deadly virus has created havoc and brought untold misery in terms of mortality. The pandemic refuses to show any obvious or even subtle signs of abating as yet, nor is there any antidote in the form of either a drug or a credible vaccine available at present to contain the proliferation of the virus. It is, therefore, pertinent to resort to the exercise of preventive measures as propounded by the various world agencies, like World Health Organisation. The obtaining scenario warrants that we at least assess for ourselves as to the timelines when the virus is expected to be easing out or mutating, gradually or otherwise following a predictable pattern, if at all. It would be tempting to turn this bane into a boon of 
sort to contemplate the possible ways including exploring mathematical modelling techniques to investigate if some tangible predictions can actually be made as to when the flex, the substantial reduction in the number of COVID-19 positive cases, and the consequential fatalities would occur. This would certainly go a long way in enhancing the psychological, albeit mental preparedness of the public at large to combat the virus effectively and to develop a pragmatic perspective to tackle this menace rationally. Dwelling on this motivation, we intend to make a modest attempt to address some of these relevant issues within the general framework of a suitable mathematical model by confronting its formulations with the currently available observational data borrowed from the governmental agencies and various other available sources.

\section{Model Prescriptions}

The essential ingredients of the mathematical formulation of a model to incorporate the patterns of the spread of the Covid-19 virus can be variously comprehended by invoking the concepts of probability, particularly those that are widely accepted and extensively used to account for the current situations. One such obvious choice is to consider the Gaussian (also known as normal) probability distribution functions to evolve a comprehensive theoretical model by virtue of which the progression of the virus could be reasonably well understood and monitored. The generalised Gauss error function, embodying the above requirements, appears to be a strong candidate to try its efficacy to fulfil the stated pursuit. We shall, therefore, endeavour to use this parametrised Gauss function to seek predictions of the dates when the flex, substantial reduction in the number of cases, and above all, the fatalities would occur.

In this context, we shall first take a quick look at the various efforts devoted in this direction. Various data driven mathematical models (SIR Model, SEIR model, SEIRU model, Bailey's model etc.) have been used recently to analyse the COVID-19 pandemic in different countries of the world and predict the number of confirmed positive cases and fatalities for future $[2,3,4,5,6]$. Some of these models and their predictions are outlined in a recent paper by Nin Wang et al [2]. Ignazio Ciufolini and Antonio Paolozzi [1] fitted the COVID-19 data using Gauss error function to predict the date of flex as well as the date of substantial reduction in the number of confirmed positive COVID-19 cases in Italy and China.

We follow the approach of Ciufolini and Paolozzi [1] and have fitted the number of cumulative positive infectious cases (I), to a cumulative distribution function (CDF) of the type of a Gauss error function (GEF) (with four adjustable parameters) of the type

$$
a+b \text { erf }(c x-d)
$$

that is the integral of a normal Gaussian distribution. The COVID-19 data for the cumulative number of positive infectious cases for the state of Maharashtra, the second most populous state of India, having a population of nearly 123 million people, has been taken from the website covid19India.org and Aarogya Setu App [6] of the Government of India. Using this approach, we calculate the dates of flex for the cumulative positive COVID-19 infectious cases (I), and for the fatalities which occurred in the state of Maharashtra. We have also calculated the approximate dates on which sufficient reduction in the number 
of confirmed positive cases (to approximately 1000) and in fatalities (to about 34) could be expected based on this model.

\section{Fit of Cumulative Positive Cases of COVID-19 in Maharashtra}

Following Ciufolini and Paolozzi [1], we approximated the number of cumulative positive infectious cases (I), to a cumulative distribution function (CDF) of the type of a Gauss error function (GEF) with four parameters, of the type

$$
a+b \text { erf }(c x-d)
$$

that is the integral of a normal Gaussian distribution. The fit of the observed data with the Gauss error function is shown in figure (1) below.

The date of flex, where the second derivative of the l-t curve becomes negative, i.e., the date from which the rate of growth of the number of infections starts decreasing using the fit has been found, using the data for the number, I, from the above sources and is found to be September 26, 2020.

The date of flex was calculated using the data for the periods from April 1 to August 11 (both dates included), then from April 1 to August 12 and so on, upto the data from April 1 to August 24 (both dates included). As the final date of analysis is increased, the date of flex quasi-oscillates. We thus obtained 14 different values for the date of flex. The date of flex from this analysis is estimated to be September 26, 2020. The standard deviation of these 14 points has a 1-sigma value of 1 day. The 2-sigma uncertainty and the 3-sigma uncertainties in the date of flex are thus 2 days and 3 days respectively.

The date of flex was also estimated by plotting the graph of the second derivative of I (GEF values, obtained numerically) versus $t$ (measured in days) and was found to be September 25, 2020 (in agreement with the above value) as can be seen from the graph below Figure (2).

The variation of the daily new cases with time is shown in the graph below and is seen to peak around the same time, after which it starts decreasing.

The values of the parameters $a, b, c, d$ in the Gauss error function for positive COVID-19 cases as on August 24, 2020 are as follows :

\begin{tabular}{rr}
1080780 & $\mathrm{a}$ \\
1085002 & $\mathrm{~b}$ \\
0.011758 & $\mathrm{c}$ \\
\hline 2.042767 & $\mathrm{~d}$
\end{tabular}


Using this fit of the above data within the prescriptions of the Gauss error function, we also calculated the date from which the increase in the number of daily cumulative positive COVID -19 cases is likely to be sufficiently reduced in Maharashtra to, say around 1000 cases. This date was calculated using the data spanning the period from April 1 to August 11 (both dates included), then from April 1 to August 12 and so on, up until from April 1 to August 24 (both days included). We thus found 14 different values for the date of sufficient reduction in the number of daily cumulative positive cases to approximately 1000 , with a standard deviation of 1 day. This date was found to be around February 7, 2021.

We then carried out a similar analysis using the data on cumulative number of fatalities due to COVID19, again taking the data from the Websites of covid19India.org, and the Aarogya Setu App of the Government of India [6].

The values of the parameters a, b, c and d for fatalities in the Gauss error function are

\begin{tabular}{ll}
16947.59 & $\mathrm{a}$ \\
17062.72 & $\mathrm{~b}$ \\
\hline 0.014982 & $\mathrm{C}$ \\
1.910964 & $\mathrm{~d}$
\end{tabular}

The variation of the daily cumulative fatalities cases with time is shown in Figure (4) below.

The date of flex for the fatalities was calculated using the data for the periods from April 1 to August 11 (both dates included), then from April 1 to August 12 and so on, up until from April 1 to August 24 (both dates included). As the final date of analysis is increased, the date of flex oscillates with a much larger amplitude than in the case of infections. We thus obtained 14 different values for the date of flex for the fatalities. The date of flex for the fatalities, where the second derivative of the F-t curve becomes negative, i.e., the date from which the rate of growth of the number of fatalities starts decreasing using the fit has been found, using the data for the number F, from the above sources and is found to be August 8,2020 .

The standard deviation of these 14 points has a large 1-sigma value of 6 days. The 2-sigma uncertainty and the 3-sigma uncertainties in the date of flex for fatalities are thus 12 days and 18 days, respectively. Thus, there is a large variation in the dates of flex for the fatalities, unlike in the case of COVID-19 positive cases, where the date more or less converges to a point as the period of analysis is increased. (The date of flex may shift ahead to a further date as more data is included in the analysis as indicated by the trend so far).

The variation of the second derivative of the number of fatalities with time is shown in Figure 5.

As in the case of number of infections, we likewise calculate the date at which the number of fatalities in Maharashtra is likely to reduce to approximately 34 (in proportion to the reduction in the number of daily increase in new positive cases to 1000). This is likely to happen on approximately November 2, 2020. This calculation was also done using the data from April 1 to August 11, Then from April 1 to August 12, 
and so on and finally from April 1 to August 24. The standard deviation of these 14 results is 11 days, implying a large 3 -sigma uncertainty of 33 days for this date.

It is pertinent to point out here that these predictions are statistical in nature and the actual dates of these events will depend on how seriously the COVID-19 protocols of wearing masks, maintaining social distancing, etc. are followed by the public and what other guidelines or relaxations in the movement and assembly of people are introduced by the Central and local Government bodies. Let us hope that a credible vaccine or some other antidote in the form of a suitable drug becomes available soon so as to effectively contain the proliferation of the virus and the situation returns to normal soon.

\section{Declarations}

Acknowledgement The author would like to express his sincere thanks to Dr. D. Parashar for his critical reading of the manuscript and for valuable suggestions.

Competing interests. The author declares no competing interests.

\section{References}

1. Mathematical Prediction of the time evolution of the COVID-19 pandemic in Italy by a Gauss error function and Monte Carlo simulations, Eur. Phys. J. Plus (2020) 135:355

2. An evaluation of the Mathematical Models for the Outbreak of COVID-19, Ning Wang,Yuting Fu,Hu Zhang, Huipeng Shi, Precision Clinical Medicine,Voume 3, Issue 2,June 2020,pages 85-93.

3. Covid-19 Predictions Using a Gauss Model Based on Data from April 2, Janik Schuttler, Reinhard Schlickeiser, Frank Schlickeiser, and Martin Kroger, Physics 2020,2, 197,212; doi: 10.3390/physics 2020013

4. Application of Mathematical Modelling in Public Health Decision Making pertaining to control of COVID-19 Pandemic in India, Anil Kumar and Rupali Roy, Special Issue on SARS-Cov-2 (COVID-19) Epidemiology International, Volume 5, Issue 2 - 2020 Pg. No. 23 - 26.

5. Mathematical Modelling and the transmission dynamics in predicting the COVID-19 - What next in combating the pandemic, A. Anirudh, Infectious Disease Modelling, Volume 5, 2020, pages 366-374.

6. Mathematical Model Based COVID-19 Prediction in India and in Different States, Jaspreet Singh, P. K. Ahluwalia and Ashok Kumar, medRxiv Preprint, 18 May 2020.

7. covid19India.org; (https://www.mygov.in /covid-19/)

\section{Figures}




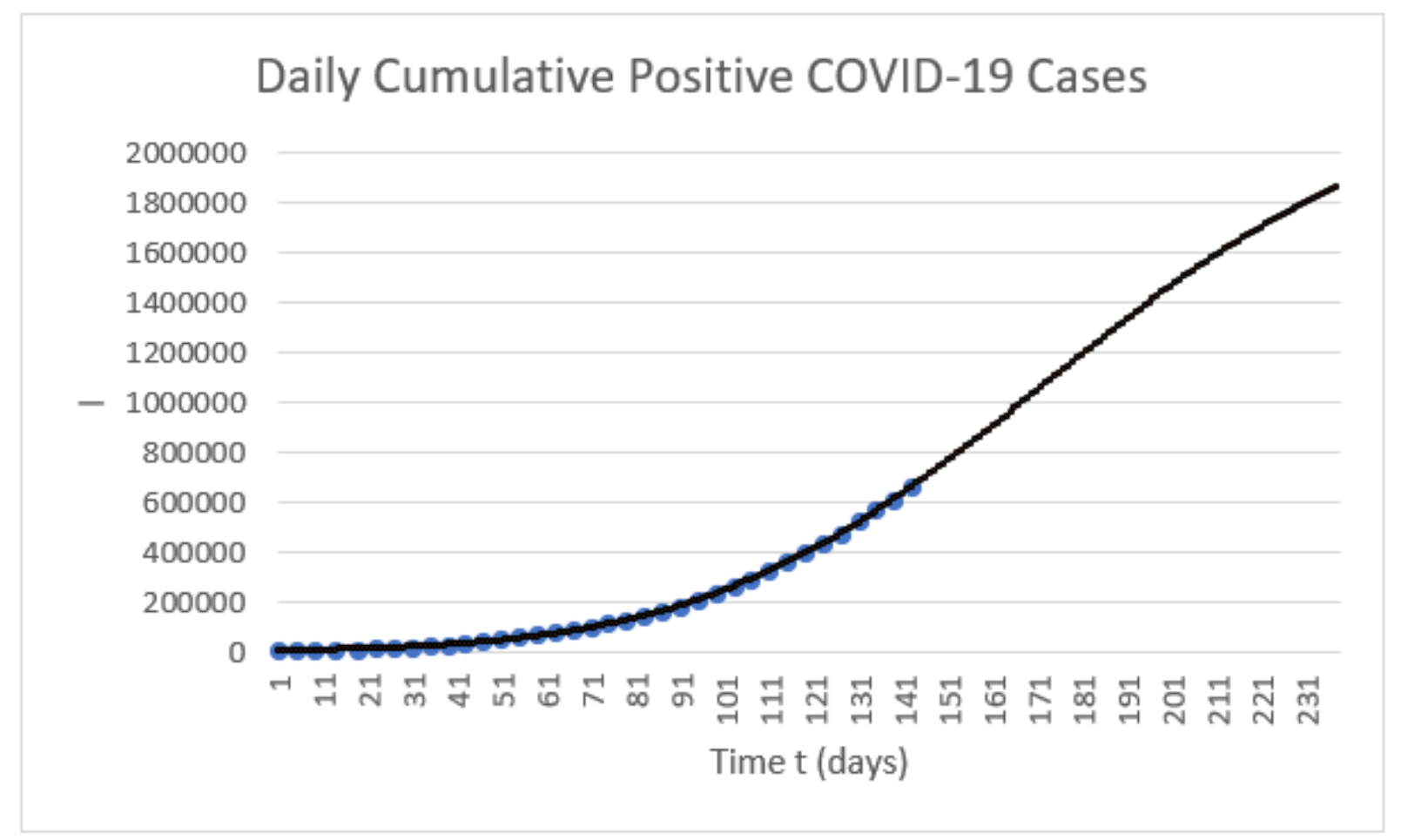

\section{Figure 1}

Daily Cumulative Infections (i) in Maharashtra as a function of time (days) from April 1 to August 24, 2020. The solid black curve denotes the values calculated using the Gauss error function. The dots denote the observed values

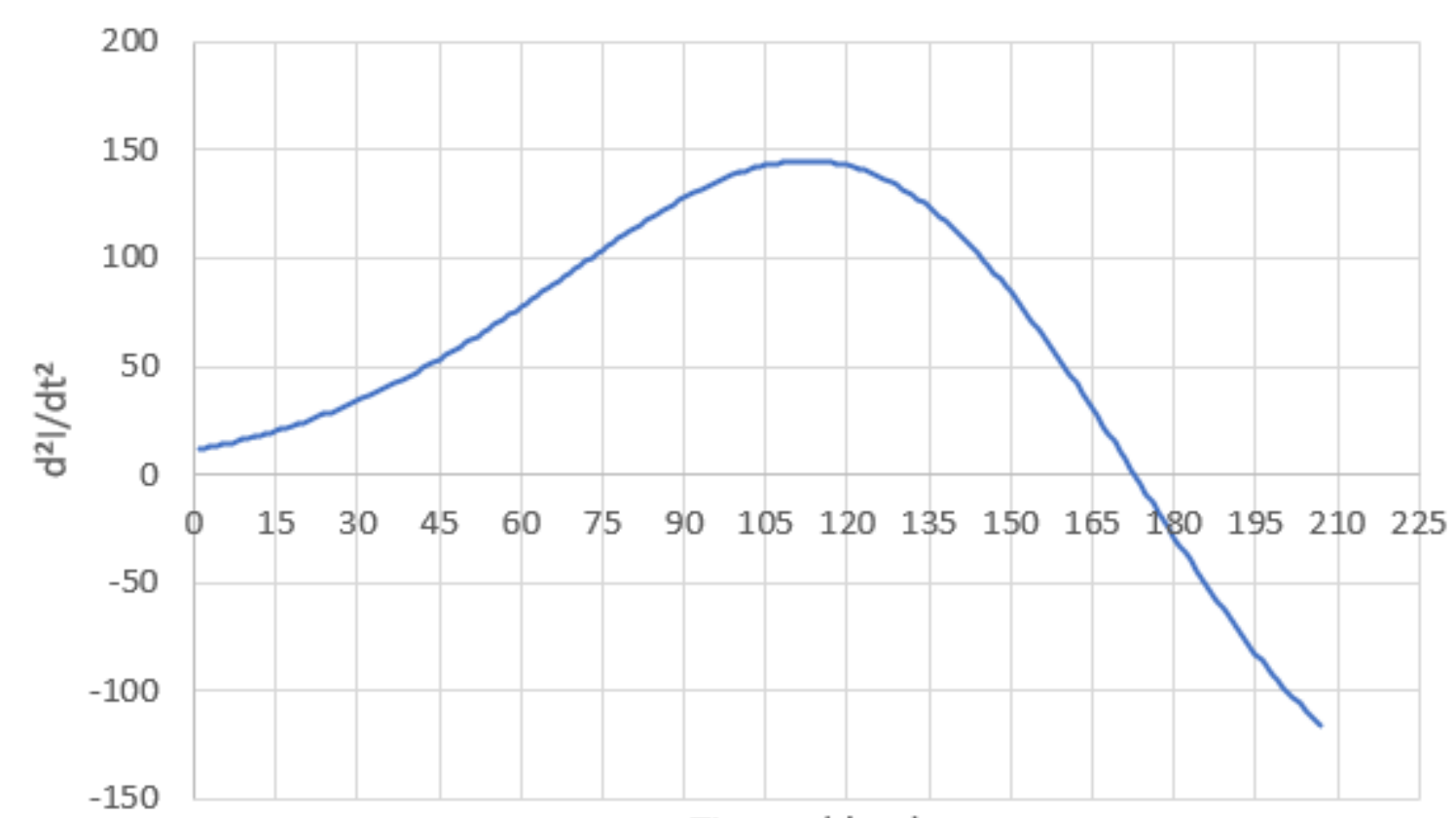

Time, $\mathrm{t}$ (days)

Figure 2 
Variation of d21/dt 2 with time. The second derivative becomes negative on day 175 starting from April 1 , 2020

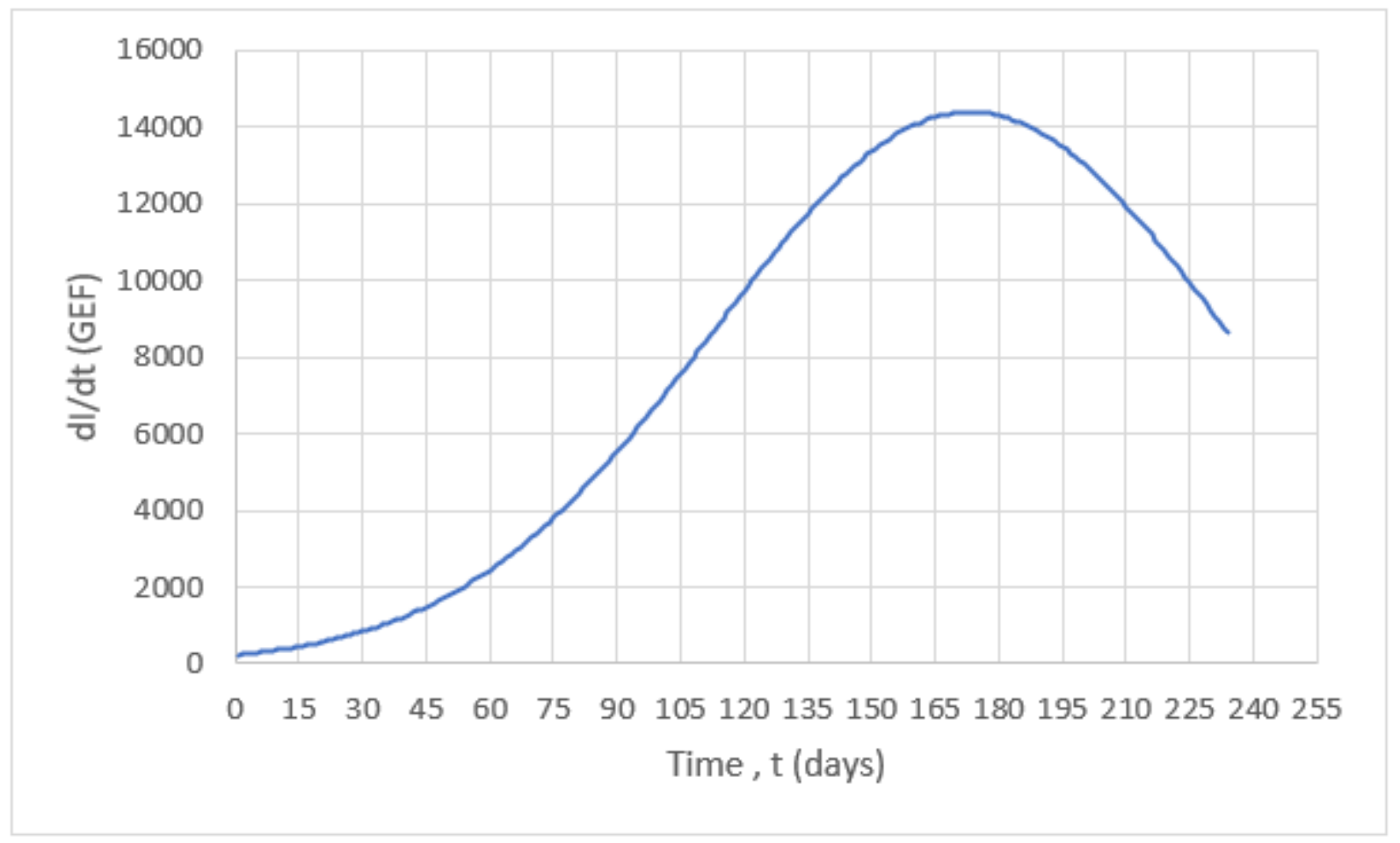

\section{Figure 3}

Variation of $\mathrm{dl} / \mathrm{dt}$ with time. The peak occurs at $\mathrm{t}=175$ days from April 2 (September 25)

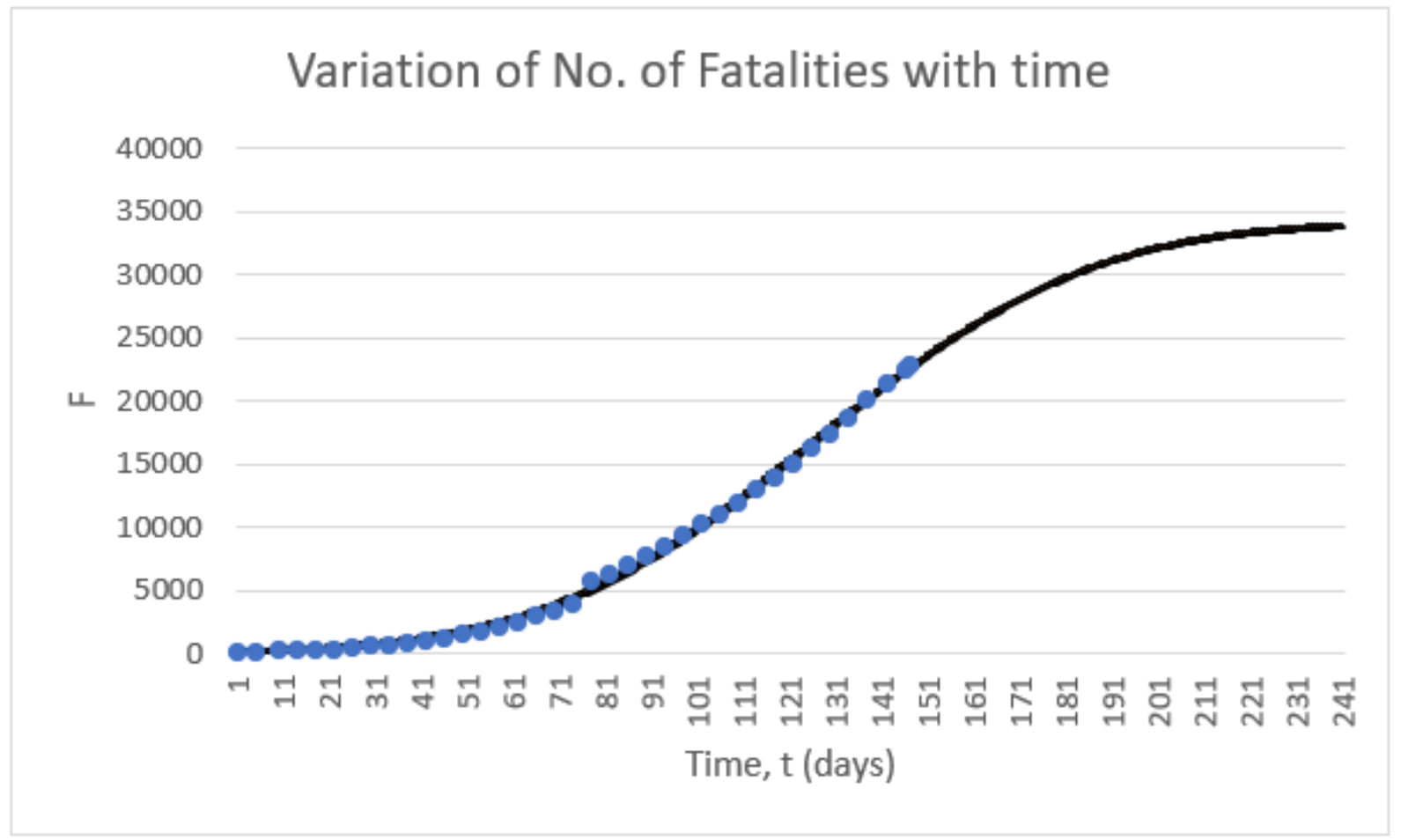

Figure 4 
Variation of number of Fatalities with time. The solid line represents the values calculated using the Gauss error function. The dots denote the observed values.

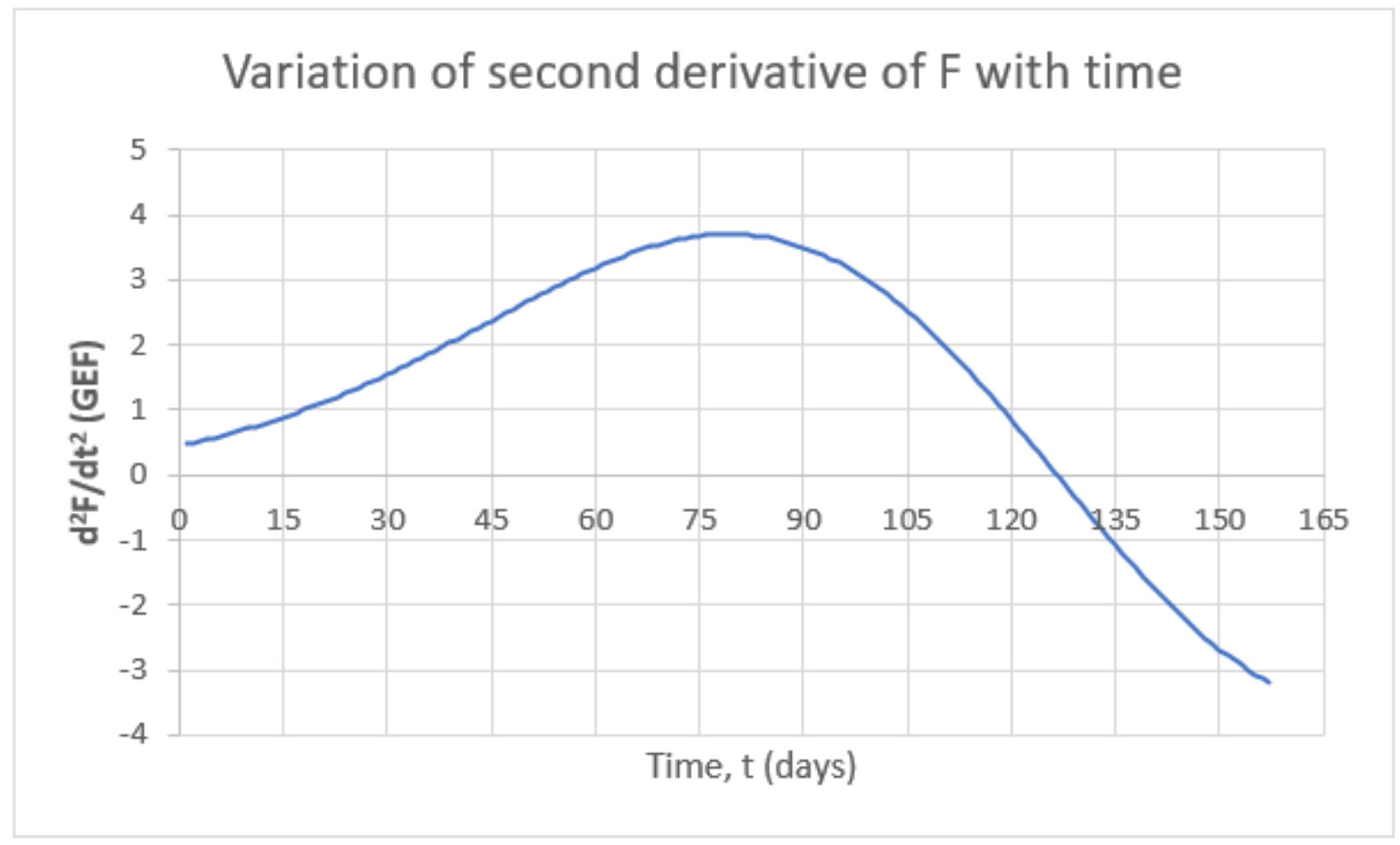

Figure 5

Variation of d2F/dt2 (GEF values) with time from April 1 to August 24. The second derivative becomes negative on day 130 starting from April 3, 2020 